\section{A Conrse of afentures}

\section{URINE， URINARY DEPOSITS, AND CALCULI.}

DELIVERED AT THE PATHOLOGICAL LABORATORY, DURING THE SESSION 1857-58.

\author{
BY
}

LIONEL BEALE, M.B., F.R.C.P., F.R.S.,

PHYSICLN TO kING'S COLLEGE HOSPITAL; PROFESSOR OF PHYSIOLOGY AND OF GENERAL AND MORDID ANATOMY IN KING'S COLLEGE, LONDON; ETC.

\section{LECTURE VII}

Urine in Disease. Insoluble Substances in Urine. 1. Float ing on the Surface, or suspended through the Fluid. Urisaky Deposits. 2. Light and Flocculent Deposits. 3. Dense and Opaque Deposits. 4. Granular and Crystalline Deposits. Examination and Preservation of Urinary DEPosits. Collecting Urine for Microscopical Examination. Period when the Urine should be examined. Removal of the Deposit from the Vessel containing it. Of Collecting a very small quantity of a Deposit from Fluid. Magnifying Power's required in the Examination of Urine. Of the Chemical Examination of Urinary Deposits. Examination of the Deposit in the Microscope. Of Placing the Deposit in the Preservative Fluid. Refractive Power of the Medium in which Deposits are mounted. Media in which Urinary Deposits may be preserved. Of Keeping the Deposit for subsequent Inquiries. Of Pre serving Deposits permanently. Mucus, Epithelium, Fungi, and Vegetable Growths. Spermatozoa. Casts. Pus. Phosphates, Urates, Blood-Corpuscles, Uric Acid, Cystine, Oxalate of Lime. On Preserving Crystalline Substances which are more or less Soluble in Water. Of Extraneous Matters of Accidentai Prfsence. Larva of the Blow. fly. Hair. Cotton and Flax Fibres. Portions of Feathers. Silk. Fibres of Deal from the Floor. Starch.Granules. Portions of Tea-Leaves. Milk. Sputum. Epithelium from the Houth. Vomit.

[Continued from $p .720$.

On the Preservation of Urinary Deposits as Permanent Microscopic; ObJects.

A DESIRE has been generally expressed that a series of the most important urinary deposits should be kept for sale, so that practitioners might have an opportunity of readily obtaining named specimens, with which the deposits that from time to time fall under their notice might be compared, and their nature recognised. Persons who prepare and sell microscopic objects have found great difficulty in preserving urinary deposits satisfactorily; and many specimens which have been pur. chased have been found to lose their characters after a few months, and have soon become quite useless objects. Feeling strongly the real practical value of preparations of this kind, it seems to me very desirable that a few rules with regard to the preservation of urinary deposits should be laid down; and I therefore propose to allude briefly to the different plans which I have found to succeed best. I hope that, shortly, there will be no difficulty in obtaining series of well-mounted and illustrative specimens.* At the same time, any one attending hospital practice, who has a little time at his disposal, can, without much trouble, prepare such preparations for himself.

The different characters of urinary deposits render necessar different plans of preservation. It is, therefore, desirable to consider the nature of the deposit before we attempt to preserve it. Some deposits may be preserved $d r y$, others may be mounted in Canada balsam. A certain number exhibit their characters very well if preserved in glycerine, while many can only be kept in certain aqueous fluids.

Of placing the Deposit in the Preservative Fluid. After the deposit has been allowed to settle in a conical glass, the supernatant fluid is to be poured off; and if it is to be mounted in fluid, a quantity of the preservative solution, equal in bulk to the urine and deposit that remain, is to be added. After the deposit has again settled, the fluid is to be poured off and

* Specimens of urinary deposits may be obtained of Messrs. Smith and Beck, Coleman Street, City; Mr. Tennant, 149, Strand; and Mr. Matthews, surgical instrument maker, Carey Street, Liucoln's Inn Fields. replaced with an equal portion of fresh preservative solution. In this way the deposit is washed clean, and properly impregnated with the preservative fluid.

When preparations are to be preserved in a fluid medium, a small shallow water-tight cell is to be used. The specimen and its preservative fluid being placed in the cell, the thin glass is applied, and the cover cemented in its place with the aid of Brunswick black or other cement. (How to Work with the Microscope.) In washing urinary deposits prior to mounting them, it is often necessary to add some compound to the water used for this purpose, in which they are known to be insoluble; and sometimes it is desirable to add some substance to increase the density of the fluid; for which purpose, certain salts, syrup, or glycerine, may be employed, according to circumstances. Many deposits, although soluble to some extent in pure water, are quite insoluble in a weak acid; others are insoluble in a weak alkali or in certain saline solutions. Again, it is sometimes desirable to separate certain substances in the deposit from others, and this may be effected by special chemical solutions which have the power of acting on the one and not upon the other; or, in cases where one is more dense than the other, by agitating the deposit with water, and, after allowing time for the heavier one to settle, pouring off the lighter one into another vessel, to subside there. From this, it may be collected in the usual way.

If the preparation is to be preserved as a dry object, water is to be added in the first place; and a portion of the deposit, which has thus been carefully washed, is to be removed with the aid of a pipette to the glass-slide, and the fluid allowed to evaporate, the whole being covered by a bell-jar, and placed over a dish of strong sulphuric acid. When dry, it is to be protected from dust by a thin glass cover. The glass cover is easily prevented from pressing upon the preparation by inter. posing a thin piece of paper or cardboard; or a thin India. rubber ring, which may be easily fixed to the glass-slide and thin glass cover, by a little gum made into a thick paste with whiting, may be used.

If the specimen is to be mounted in Canada balsam or turpentine, it is to be dried in the manner just described, warmed slightly, wetted with the balsam, and mounted with the usual precautions. (How to Work with the Microscope.)

Refractive Power of the Medium in which Deposits are Mounted. The appearance of objects in the microscope depends very much upon the medium in which they are im. mersed; and many structures are so altered in their character by different media, that they would hardly be recognised as the same object. It may be said generally, that the darker the object, and the more dense its structure, the higher should be the refractive power of the medium in which it is mountedthus the dark coloured uric acid, or the thick spherical crystals of carbonate of lime, and the dumb-bells of oxulate of lime, exhibit their structure to the greatest advantage when mounted in the highly refracting Canada balsam, or in strong syrup or glycerine, while the beautifully transparent octohedra of oxalate of lime would be scarcely visible in these media, and require to be mounted in an aqueous fluid which possesses a lower degree of refractive power. Many of these objects, when mounted dry, appear quite dark, and scarcely exhibit any structure at all, in consequence of great difference in the refracting power of their substance, and the air by which they are surrounded. From what I have said, it will be evident how im. portant it is to examine the same object in different media-in fact, it is quite impossible to form an idea of the real structure of many specimens, without proceeding in this manner. (How to Work with the Microscope, p. 59; and The Microscope in its Application to Practical Medicine, second edition, sects. 74 , 89 , and 90. )

Media in which Urinary Deposits may be preserved. Urinary deposits may be mounted in air, in turpentine, oil, or Canada balsam; in glycerine, in gelatine and glycerine, in solution of naphtha and creasote, in certain saline solutions, in weak spirit, and in some other aqueous solutions, which will be alluded to. The "glycerine" which I use is Price's patent glycerine, diluted with water.

The composition of the naphtha and creasote fluid, above referred to, is as follows :-

Solution of Naphtha and Creasote.

Creasote..................... 3 drachms.

Wood naphtha $. . \ldots \ldots \ldots \ldots \ldots, 6,6$ ounces.

Distilled water $. . \ldots \ldots \ldots \ldots \ldots \ldots .6 \pm$ ounces.

Chalk, as much as may be necessary.

Mix first the naphtha and creasote, then add as much pre- 
pared chalk as may be sufficient to form a smooth thick paste; afterwards add, very gradually, a small quantity of the water, which must be well mixed with the other ingredients in mortar. Add two or three small lumps of camphor, and allow the mixture to stand in a lightly covered vessel for a fortnight or three weeks, with occasional stirring. The almost clear supernatant fluid may then be poured off and filtered, if neces sary. It should be kept in well corked or stoppered bottles.

Of keeping the Urinary Deposit for subsequent inquiries. I cases where it is desirable to retain a certain quantity of the deposit in the preservative solution for subsequent examination, or for the purpose of making more preparations, it should be kept in a small glass tube, with a tight fitting cork, and carefully labelled. Most urinary deposits may be kept for a longer time in this manner, than mounted in thin cells. I propose now to describe briefly the various plans adapted for the preservation of urinary deposits which I have found to succeed best.

\section{First Class Urinary Deposits.}

Mucus. It is very difficult to preserve the character of the so-called " mucus corpuscles", or imperfectly formed epithelial cells, nuclei, and granules, which constitute the slight flocculent deposit met with in healthy urine, and termed "mucus". The naphtha and creasote solution is best adapted for the purpose, and it is desirable to place the specimen in a cell about the twentieth of an inch in depth.

Epithelium. The different varieties of epithelium are easily preserved, although, after the lapse of some time, minute oil globules make their appearance in them. They may be kept in naphtha and creasote fluid, to which one-fourth of its bulk of glycerine has been added. It is well to put up specimens of epithelium from the urethra, bladder, ureter, and pelvis of the kidney, removed from the organs of a healthy man who has been killed accidentally. They should be mounted in very thin cells. Specimens of the epithelium from the vagina, which can generally be obtained from the urine of females, should also be preserved.

Vegetable Growths: Fungi. I have found that fungi may be preserved most satisfactorily in glycerine, for although they appear somewhat more transparent in this fluid than in urine, they preserve their general character better than when im mersed in other preservative fluids. It is necessary to add weak glycerine in the first instance, and to increase the strength gradually, otherwise the fungi become collapsed, owing to the great density of the strong solution. A solution composed of equal parts of water and Price's glycerine is sufficiently strong to preserve fungi. I have not been able to preserve specimens of sarcinæ which I have met with on two or three occasions in the urine, probably in consequence of their extreme delicacy. The sarcinæ which are from time to time met with in vomit keep perfectly well, and preserve their recent characters in glycerine.

Spermatozoa are sometimes mounted in the dry way; but although their general form is preserved, their refractive power and transparent appearance are so different from what is observed when they are immersed in urine, that little is gained from such preparations. Spermatozoa keep very well in glycerine, although they appear rather more faint than in an aqueous fluid. They should be examined with the eighth of an inch object-glass ( $X$ about 400 ); but when the eye of the ob. server has become familiar with the general appearances, they may be readily recognised with a quarter of an inch object. glass ( $x$ about 200 ).

Casts. It is not difficult to preserve the character of some varieties of casts. The transparent casts often become covered with numerous minute granules and oil globules, and their character much altered. Granular casts and epithelial casts often keep very well in the naphtha and creasote solution; but altogether I prefer glycerine, with one-third part of water. Although in many instances the cells they contain are altered, and oil globules appear much more transparent than when in urine, this alteration in character may be easily allowed for. I have some specimens of large waxy casts and epithelial casts which have been kept in the naphtha and creasote solution for upwards of seven years, and still preserve their characters well. The specimens in glycerine, of course, keep admirably. Some casts may also be preserved in gelatine and glycerine, care being taken that the mixture is not made too hot.

\section{Second Class of Urinary Deposits.}

Pus. Recent specimens of pus may be so readily obtained that it is hardly necessary to attempt to preserve the corpuscles permanently. Their characters alter so much in all the pre- servative fluids that I have tried, that after they have been put up for some time, it would be difficult to recognise the nature of the preparation.*

Phosphates. The phosphate of lime, in its amorphous form, in globules, and minute dumb-bells, is easily preserved in weak spirit, naphtha and creasote fluid, or glycerine; but the character of the crystals of the triple, or ammoniaco-magnesian phosphate, could not be retained in this solution. As is well known, this salt is quite insoluble in solutions of ammoniacal salts, and these make the best preservative solutions for it. Crystals of triple phosphate may be liept for any length of time, with their smooth surfaces and their lustre unimpaired, in distilled water, to which a little chloride of ammonium has been added. Phosphate of lime and the stellar form of triple phosphate may be dried carefully, and mounted in Canada balsam; but, of course, the appearance of the crystals is a good deal altered.

Urates. As the urates are so commonly met with, and as they are generally deposited in the form of granules, there is scarcely any need of mounting them as permanent objects. If desired, however, deposits of this kind may be preserved by adding a little naphtha and creasote fluid to the deposit, which should be left in it for a considerable time before it is put up. Urates which crystallise in small spherical masses, as often occur in the urine of children, and more rareiy in irregularly branched processes, may be preserved very well in Canada balsam, or, if preferred, they may be kept in the naphtha and creasote fluid.

\section{Third Class of Urinary Deposits.}

Blood Corpuscles become more or less altered in most preservative fluids. I think that those which I have mounted in glycerine (one part glycerine to two parts of water) have undergone the least change.

Uric Acid Crystals are easily preserved as permanent objects. The usual plan is to mount them in Canada balsam. They should be washed, in the first instance, with a little water, to which a few drops of acetic acid have been added. When pretty clean, they may be placed upon a glass slide, with the aid of a pipette, and the greater quantity of the fluid absorbed with a small piece of bibulous paper. After the crystals have been properly arranged on the slide with a needle, they may be dried, by exposure under a bell jar over a dish containing sulphuric acid. When quite dry, they may be moistened with a drop of turpentine, and mounted in Canada balsam. In this operation, a very slight heat should be employed, otherwise the crystals will become cracked in all directions, and more or less opaque. Uric acid crystals, as a general rule, do not keep well in glycerine. In cases where we wish to preserve other substances in the deposit as well as uric acid crystals, the naphtha and creasote fluid will be found to answer very well. I have some preparations mounted in this manner, which were put up six or seven years ago.

Cystine. Crystals of cystine may be preserved in Canada balsam, the same care being taken in mounting them as men tioned under uric acid, or they may be kept very well in distilled water, or in the naphtha and creasote fluid, to which a little acetic acid has been added.

Oxalate of Lime. Both the octohedra and dumb-bells may be preserved in glycerine; but the former look very transparent in that fluid. The dumb-bells may also be mounted in Canada balsam, but in this medium the octohedra are almost invisible. When required for polarising, they should be put up in balsam. The dumb.bells keep very well in glycerine.

On Preserving Crystalline Compounds obtained from Urine. It is exceedingly difficult to preserve many of the crystalline substances obtained from urine in a moist state; but sereral of them form beautiful microscopic objects when carefully dried. Urea, nitrate of urea, oxalate of urea, creatine, creati nine, alloxan, hippuric acid, murexid, and many others, may be kept as permanent objects in this manner. In order to prepare them, it is better to cause them to crystallise upon a glass slide; allow the mother liquor to drain of, and immediately place the slide under a bell-jar over sulphuric acid. Sometimes the crystals may be made in a small evaporating basin, and when drained and dried, a portion of them may be removed to a glass cell, and covered with a piece of thin glass to exclude the dust. Many crystals may be examined and preserved for a considerable time in their own mother liquor, especially when they are very slightly soluble in fluid; but, as a general rule,

* Dr. Andrew Clarke speaks highly of some fluids, containing bichloride of mercury and arsenious acid, which he has prepared. (The Microscope in its Application to Practieal Aledicine, second edition, p. 237 , note. 
this plan does not answer very satisfactorily, for, independently of the escape of the fluid from the edges of the cell, a few of the largest crystals grow still larger at the expense of the smaller ones, and the beauty of the specimen is destroyed. The different forms of these crystals, as they appear in the microscope, are given in the Illustrations of Urine, Urinary Deposits, and Calculi. (Urine, plates I to Ix.) See also The Microscope in its Application to Practical MIedicine, chapter ix, page 292 .

[To be continued.]

\section{OAtrinal Communications.}

\section{WARTY VEGETATIONS NEAR THE ANUS, AND THEIR TREATMENT. \\ By Tromas T. Griffith, Esq., Wrexham.}

Is a clinical lecture by M. Nélaton, reported in the Journal de Méllecine et de Chirurgic Pratiques for February last; he relates the case of a girl about 14 years of age, who was the subject of a very extensive growth of warty vegetations, seated partly within the anal orifice and partly around it, and attached to the skin by a membranous connexion. Its surface was granular, crackel, and secreted a sero-sanguinolent fluid. The anus seemed lost in the enormous cauliflower-looking mass, but still it retained its normal diameter. The disease might have been supposed to be syphilitic; but, after a careful consideration of all the special circumstances of the case, M. Nélaton decided against such an opinion; he also expressed doubts as to the disease commonly having such an origin. He had often seen patients labouring under it who had had no venereal taint, and others who never had had sexual intercourse; though he believes that patients actually syphilitic, or those frequently liable to it, are more likely to become the subjects of this morbid growth than others. He viewed it as an accident purely local, resulting from varied and combined causes. $\mathrm{He}$ advises caution in receiving the patient's own statements, and thinks it best to give a decided opinion only when it can be based on authentic facts. His treatment is entirely local. His patient was chloroformed, and the mass was removed by a ligature of annealed iron drawn so tight as to act as an écraseur. The raw surfaces were painted with perchloruret of iron, which acted as a styptic; and mild cauterisation, etc. For a few days afterwards, the parts were bathed with camphorated spirits. The cure was speedy and complete, and no fears of the return of the disease were felt.

My object in giving an abridged account of this case is to in. troduce one similar to it in its leading particulars.

CASE. Mr. J., aged 35, married, of good general health, about two years ago was aware of small warty growths near the anus, which gradually increased, extending along the opposed surfaces of the nates. 'They acquired in time a considerable size, and were occasionally sharply painful and inconvenient. Little or no discharge formed on the surface.

I saw the patient in January last, and advised the removal of the mass, which now occupied a space of about six square inches. The anus could only be discovered by the forcible separation of the nates. The growth seemed to consist of warty vegetations, each of which had its own independent root in the skin; but, as they grew close and parallel to each other, they formed masses most like the cauliflower, with granular cracked surfaces of a brownish colour. They showed no disposition to bleed on being touched, though doing this caused exquisite pain, which, I believe, arose from sensitiveness in the subjacent skin, and not from the growth itself.

The patient was placed as for lithotomy, and I removed, partly with a scalpel, but more readily with strong scissors curved on the flat, nearly the whole mass, when the patient felt very ill; and as he became rather faint, and the bleeding was free, I left a portion immediately around the anus, and stopped the bleeding by the free use of tincture of sesquichloride of iron. It, however, returned to some extent in the night. The next day, the patient was comparatively well, walked out, and in a few days resumed his professional duties. The portions that remained were, after a few months, entirely removed, and the patient was at once restored to a state of ease and comfort that he had not felt for a length of time.
The skin operated on very rapidly regained its usual healthy state and appearance.

In this case, there were no grounds for suspecting any specific taint, as the patient never had syphilis or gonorrhœa in any form.

[It may not be generally known that caustic marks on the honds, of some days standing, may be removed by a strong solution of iodide of potassium rubbed well over the surface; and, by applying afterwards a little liquor potassæ, the skin is restored to its usual soft smooth state.]

\section{A N O B S C UR E C A S E \\ By W. Sankey, Esq., Dover.}

Wr. Henry Bew, aged 13 years, applied to me on the 4 th September 1860, with the following symptoms: excessive weakness, emaciation, constant thirst, feeble pulse, coated tongue, and dry hands. He complained of no pain. His mother stated that from his birth he had been healthy and strong, and fond of active pursuits; but that within the last three weeks he bad rapidly lost flesh and strength. On inquiry, I found that he passed a large quantity of limpid urine, and wetted his bed every night.

I prescribed three grains of hydrargyrum cum cretâ with two grains and a half of Dover's powder at night, and a mixture of nitric and hydrochloric acids with quinine thrice daily. I requested to have some of his urine for analysis at his next visit.

September 7th. I received a message to visit him on that or the following day, as he seemed much worse. About nine in the evening, a second message came; and I lost no time in going to see him at about three miles distance.

On my arrival, I found him rapidly sinking. The extremities were cold; the pulse was scarcely perceptible; he was perfectly conscious, complaining of nothing but thirst. Death did not take place till eight hours afterwards. I applied for permission to make a post morten examination, but did not obtain it. Leave was given to pass a catheter with a view to obtain some urine for analysis; but the bladder was found empty.

\section{Cramsactions of 通xancthes.}

\section{CAMBRIDGE AND FUNTINGDON BRANCH.}

$\triangle$ CASE OF EPILEPST, WITH SOME UNCOMMON SYMPTOMS : AND A COMMENTARY THEREON.

By G. E. Paget, M.D., F.R.C.P., Cambridge. [Read at Cambridge, July 10th, 1860.]

IT is well known that the convulsive phenomena of epileptic fits differ more or less in different patients, and that the same patient is not unfrequently subject to attacks of two kinds differing in some respects, generally in the degree of their violence. It is unnecessary for me to dilate on this fact, or describe any of the more frequent deviations from the normal type; but in the case I am about to relate, and in which the attacks are of two different kinds, the minor attacks are exceedingly peculiar. They assurne a form which $I$ never before witnessed, and which is, I believe, in the highest degree rare.

D. $\mathrm{C}$, an agricultural labourer, residing with his parents at Meldreth, aged 20, and unmarried, was admitted an in-patient at Addenbrooke's Hospital, on July 2nd, 1856. From that time until August 1858, he was almost continuously under observation, either as in- or out-patient. At first, he was under the care of Dr. Bond; afterwards, for a long period, under my care; and for a short time under that of Dr. Fisher. My notes of his case extend, in detail, with few interruptions, over two years; and on the 16th of last month, he was again (after a long interval) admitted to the Hospital, and is at the present time one of its inmates.

$\mathrm{He}$ had (and has) much of the ordinary appearance of an agricultural labourer: a robust figure, a head not ill shaped, but the occiput and nucha are large, and his forehead looks heavier than it did in 1856. His features are regular, but have a dullish grave cast. His intelligence is of an ordinary kind, 